UC-34

Issued: Ci-tober 1985

LA- - 10530-MS

$\mathrm{DE} 86 \quad 005865$

\title{
A Discussion of Material Rotation and Stress Rate
}

\author{
John K. Dienes
}

\section{DISCLAIMER}

This report was prepared as an account of work sponsored by an agency of the United States Government. Neither the United States Government nor any agency thereof, nor any of their employees, makes any warranty, express or implied, or assumes any legal liability or responsibility for the accuracy, completeness, or usefulness of any information, apparatus, product, or process disclosed, or represents that its use would not infringe privately owned rights. Reference herein to any specific commercial product, process, or service by trade name, trademark, manufacturer, or otherwise does not necessarily constitute or imply its endorsement, recommendation, or favoring by the United States Government or any agency thereof. The views and opinions of authors expressed herein do not necessarily state or reflect those of the United States Government or any agency thereof.

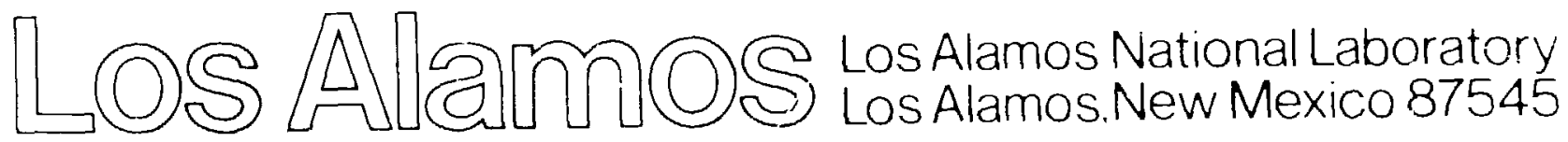




\section{CONTENTS}

ABSTRACT

I. INTRODUCTION

I I. SUMMARY OF STRESS RATE THEORY

III. DISCUSSION OF RECENT WORK

IV. CONCLUDING REMARKS

REFERENCES 


\title{
A DISCUSSIUN OF MATERIAL ROTATION AND STRESS RATE
}

\section{by}

John $K$. Dienes

\begin{abstract}
Characterization of material behavior can be divided into two parts, the analysis of deformation and the underlying physics, though these are intimately related. A significant advance in the analysis of deformation was made whon the polar decomposition theorem was introduced, making it possible to separate large deformations into a stretch and a rotalion. Consequences of the theorem affezt th ly rate processes should be characterized. In particular, rate of macerial rotation is different from voiticity, and the stress rate for finite strains is different from the usual stress rate of Zaremba, Jaumann, and Noll. It is convenient to define a strain rate that is different from the stretching that is the symmetric part of the velocity gradient. These concepts are described in detail in a 1979 paper. Various criticisms of that paper have appeared in the Journal of Applied Mechanics, which are discussed herein. To illustrate the distinction it is shown that the rate of rotation in a classical vortex does not vanish, though the vorticity is zero. It is also shown that the rate of material rotation recently computed by Nemat-Nasser, which involves an eigenvalue expansion, is equivalent to the one given in the 1979 paper, which makes use of matrix inversion, and it asseverated that the matrix inversion approach is computationally more efficient.
\end{abstract}

\section{INTRODUCTION}

Though the theory of material rotation lies only at the periphery of the theory of material behavior, it receives continuing attention [1-3] because it is a necessary ingredient of any general theory, and if not adequately treated spurious results may follow from an otherwise adequate approach. The paper of Nagtegaal and de Jong [4] presents an interesting example of how use of the traditiona! ZJN approach of Zaremba [5], Jaumann [6] and Noll [7] can lead to unrealistic behavior of the stresses at very large strains in a calculation of simple shear with kinematic hardening. Similar difficulties are illustrated in anorher example involving a simple hypoelastic material given by Dienes [8]. These 
difficulties are avoided when material deformation is treated with polar decomposition as the starting point. This was demonstrated by Dienes [8] in a paper outlining a general theory. The essential new issue in that paper, which makes calculations of an analytical or numerical character possible, is the development of a relation between rate of material rotation $\Omega$ and vorticity $W$. The general theory of stress rate is considered therein using a line of argument similar to that of Noll [7] and Green and McInnis [9]. In this discussion the relation of $\Omega$ and $W$ is further illustrated using a simplified version of the general result that is valif for plane flows. In addition, it is shown that the relation of $\Omega$ and $W$ is equivalent to one put forth by Nemat-Nasser [1] 11sing the method of spectral analys is developed by Hill [10].

It is argued by Lee [2] that the porar stress rate (this term is introduced here to describe a stress rate involving the use of polar decomposition) violates an axiom of plasticity. It is shown in this discussion, however, that the appearance of the polar stress rate in constitutive laws arises from the elastic part of the elastic-plastic flow rule, and its structure does not affect normality or the yield condition. Prager [11] discusses the theory of stress rates and argues that the ZJN stress rate has an advantage over other stress rates (such as Oldroyd's) in that the second invariant remains constant when it is used to characterize plastic flow. Prager's argument can be used equally well to support the polar stress rate. The plan of this discussion is to summarize the main results of [8], provide a new illustration of those results, and then to relate those results to recent discussions of material rotation.

\section{SUMAAR OF STRESS RATE THEORY}

The motivation for the analysis of Ref. 8 arose from the observation that two different angular rates appear in the analysis of deformation, the vorticity $W$ and the angular velocity $\Omega$. Thus, it seemed natural to inquire about the relation between them, and to determine the context in which each should be used. The analysis followed a reading of Truesdell [12] and, consequently, the notation, and in many places the terminology, follow that elegant, though paperbacked, volume. Though Nemat-Nasser [l] has maintained that it is inappropriate to modify stress rates in order to prevent "strange and unwanted results" from emerging, in fact no such ad hoc procedure was followed. The relation of $\Omega$ and $\mathrm{W}$ was obtained first, the derivatiun of stress rate followed, and then the comparison of the polar stress rate and the ZJN approximation was carried through as an 
illustration. Thus, the unrtalistic character of the ZJN approximation at large' stralns appeacel as a considquence, rather than a premise, of thr analysis of Ref. 8.

When the deformation gradient $"$ is resolved into the product VR, where " is the positive-definite spatial stertch and $k$ the naterial rotation, it is straighttorward [8] to show that the velocity yradient fo can be expressed in rither of the forn:

$$
G=n+w=b v^{-1}+v a v^{-1}
$$

whers !) i.i the stertehing, is the vorticity, and

$$
\lambda=\dot{R}_{3}{ }^{\mathrm{T}}
$$

is the cate of materel rotation. Fquation (I) can he regarded as a set af tirces linear, similtanous equations for oinvolviag $D, W$, and $V$. If at any instant the curcent configuration is taken as the reference configurition, then $\because=I$ and 1t Eollows that $\dot{y}=D$ and $\partial=w$. This, however, is not the general situation in dynamic alculations, for $V=$ I is genrally an initial condition, the reference configuration is given at a fixpd zime, and $\Omega$ and iv are different. It is in this respert that the current throry differs from Noll's $17 \mid$, for he considers the curront and reference states it-ntical in connection with stress-rats analysis. Fipution (1) can he solved for the angular rate in a convenient form by replacing $\therefore, H$, and 7 , where

$$
Z=D V-V D,
$$

by the corresponding vectors $w, w$, and $z$, for then, as shown by Dienes [9]

$$
\omega=w+a \text { or } \quad w=w+A
$$

where the vector a is given by

$$
a=(I \operatorname{tr} V-V)^{-1} z \text {. }
$$


Though in many situations the vorticity $W$ may be a good approximation to the rotation $\Omega$, it is not intvitable. For example, the simple plane vortex of elementary hydrodynamics has zero vorticity almost everywhere, but material rotation is clearly involved. To illustrate this quantitatively, the deformation can be uritten as

$$
x=r \cos \alpha, y=r \sin \alpha
$$

where

$$
\alpha=\omega t+\phi, \tan \phi=Y / X
$$

and

$$
\omega=k / r^{2}
$$

with $x$ representing the strength of rhe vortex. The deformation gradient is (ignoring the third dimension)

$$
F=\left(\begin{array}{cc}
\cos \beta+\mu \sin \alpha \cos \phi & -\sin \beta+\mu \sin \alpha \cos \phi \\
\sin \beta-\mu \cos \alpha \cos \phi & \cos \beta-\mu \cos \alpha \sin \phi
\end{array}\right)
$$

where

$$
\mu=2 \kappa t / r^{2}, \quad B=\omega t
$$

and the velocity field is given by

$$
\dot{x}=-(k / r) \sin \alpha, \dot{y}=(k / r) \cos \alpha .
$$

It follows by direct calculation that

$$
D=\omega\left(\begin{array}{cc}
\sin 2 \alpha & -\cos 2 x \\
-\cos 2 \alpha & -\sin 2 \alpha
\end{array}\right)
$$


and, of course, that the vorticity vanishes. The stretch $V$ can be evaluated by spectral analysis of $B=F F^{T}$, but the calculation can be greatly simplified by means of the relation

$$
V=\frac{B+\sqrt{I_{2}} I}{\sqrt{I_{1}+2 \sqrt{I_{2}}}}
$$

where $I_{1}$ and $I_{2}$ are the first and second invariants of $B$. This relation can be obtained Erom the Cayley-Hamilton theorem, as indicated by Marsden and Hughes $|13|$ p. 55. (Their relation contains a signerror, corrected in (13), and here we are concerned with $V$ rather than $\vec{V}$, which they call ll, but the methudolosy is theirs.) The result of this calculation is

$$
V=\frac{1}{\sqrt{4+\mu^{2}}}\left(\begin{array}{l}
2+\mu \sin 2 \alpha+\mu^{2} \sin ^{2} \alpha-\mu \cos 2 \alpha-\frac{\mu^{2}}{2} \sin \alpha \\
-\cos 2 y-\frac{\mu^{2}}{2} \sin 2 \alpha \\
2-\mu \sin 2 \alpha+\mu^{2} \cos ^{2} \alpha
\end{array}\right)
$$

ignoring, agiain, the third dinension. Then, $z$ (the spin commutator) is

$$
Z=D V-V D=-\frac{\omega \mu^{2}}{\sqrt{4+\mu^{2}}}\left(\begin{array}{cc}
0 & 1 \\
-1 & 0
\end{array}\right) \text {. }
$$

For plane flows the matrix inverison of (5) can be avoided. It is straightforward to show that the only non-zero term in $\omega$ is

$$
w_{3}=w_{3}+\frac{z_{3}}{v_{11}+\frac{v_{22}}{v_{2}}} \text {. }
$$

Since the vorticity vanishes, application of this result to vortex motion leads to 


$$
\omega_{3}=\frac{w \mu^{2}}{4+\mu^{2}} \text {. }
$$

The sign convention of Ref. 8 makes a positive rotation counterilockwise, so that (17) represents a counterclockwise rotation (provided $\omega$ is positive, indicating a clockwise vortex). This is what might be expected, for if a pine needle were to enter a clockwise vortex its outside would rotate more slowly than the inside, inducing a counterclockwise motion. For large $\mu$, the rate of rotation is $\omega$. the same as the angular rate of the material, indicating that the needle is rotating once per revolution, always tangent to a circular streamline. The total rotation, obtained by integrating (17), is less than the rotation of the vortex about its center, w, by $\pi / 2$ at late times. This reflects the fact that the angular rate is less than its steady-stace value at early times. Consicier now a constitutive relation of the form

$$
\dot{\sigma}=\psi(\sigma, D, V)
$$

involving the Cauchy stress $\sigma$ and its ordinary rate $\dot{\sigma}$, assumed given, but determined from considerations that do not involve material rotation. Such a relation inciudes viscoelastic and elastic-ideally-plastic flow rules, and can be readily generailzea to account for hardening behavior. Suppose, now, that a material characterized by (18) is undergoing a deformation that includes rotation, and assume that the rotation does not affect material behavior. (This seems quite reasonable for solids, but for gases it has been the subject of a spirited discussion by Woods $[14,15]$ and others.) Variables referred to the rotating material axes are denoted with bars. Then

$$
\bar{\sigma}=R^{T} \sigma R, \bar{V}=R^{T} V R, \bar{D}=R^{T} D R
$$

and other variables such as the back stress $\alpha$ appearing in kinematic hardening theories transform in the saue way. The constitutive law can be used without modification if material (barred) variables replace the spatial (unbarced) varia*bles in (18), so that 
This expression can be used in dynamic calculations provided that $R$ is known. If it appears desirable to use space axes and express behavior in terms of Cauchy stress, the expressions of (19) must be substituted into (20). The resulting constitutive law has the form

$$
\hat{\sigma}=\dot{\sigma}-\Omega \sigma+\sigma \Omega=\psi(s, D, V)
$$

under very general conditions for the function $\psi$, provided that the material behavior is isotropic. The polar stress rate on the left accounts for the effect of rate of material rotation on the compunents of the stress tensor, as well as for changes due to continuing deformation. If one sets $V=I$, then $?$ becomes equal to $W$ and the expression on the left becomes the ZJN approximation. In some cases the ZJN stress rate may provide a useful estimate. For large deformations, however, the ZJN approximation can lead to large errors and unstable behavior,. as indicated by Dienes [8] in an example concerning hypoelastic behavior, in another example concerning elastic-plastic kinematic hardening [16] and by Dafalias $[3]$ in an analysis of a rigid-plastic representation of kinemctic hardening.

\section{DISCUSSION OF RECENT HORK}

An alternative view has been put forth by Nemat-Nasser [1] who takes the position that "... it is not the Jaumann rate... that nught to be suspect when strange and unwanted results emerge... but rather it is the constitutive form that may not be appropriate in conjunction with the chosen flux for the intended objectives." In this view it is argued that an equation having the form of (21) can be replaced by one involving the Jaumann (ZJN) rate

$$
\sigma=\dot{\sigma}-W_{\sigma}+\sigma W=T(\sigma, D, V)
$$

in view of (4), where

$$
T=\psi+A \sigma-\sigma A
$$


Thus, changing the stress rate is considered in some sense equivalent to changing the constitutive law. However, in the derivation given in the preceeding section (which follows the same line as Noll [7] and Green and McInnis [9]) the constitutive law, characterized by the right-hand side of (18), is considered given. It can be considered given by data, a gued by plausible inference or rigorously derived by physical and mathematical arguments, but it is considered given. Then one considers how it should be generalized to account fnr material rotation, and concludes that all that is necessary, for isotropic materials, is to replace the ordinary stress rate in (18) by the polar stress rate of (21). Though (21) and (22) would be equivalent if $\psi$ and $T$ were arbitrary, such is not the case, tor $\psi$ $i=$ specified. An additive term $A \sigma-\sigma A$ in $T$ would have no meaning in terms of material response, since it contains no physical constants, nor does it reflect any information about the mechanics of material behavior.

In Ref. $l$ it is also stated that "Dienes seeks to remove it (oscillatory behavior) by using the objective stress rate $R\left(R^{T} \sigma R\right)^{*} R^{T}$ in place of $\sigma \ldots$. In fact, as mentioned above, the development of (21) for $\sigma$ was obtained by the direct argument summarized above, and was motivated only by an interest in understanding the role of material rotation in constitutive laws. The oscillatory behavior of the stresses arising from use of the ZJN approximation appeared only subsequently, and was only an incidental aspect of the example.

Nemat-Nasser goes on to provide an expression for A ( $\varepsilon$ in his notation) given by

$$
A_{j k}=\sum_{r, t} \frac{\lambda_{t}-\lambda_{r}}{\lambda_{t}+\lambda_{r}} \tilde{D}_{r t} T_{j r} T_{k t}
$$

in current notation, where

$$
\tilde{\mathrm{D}}=\mathrm{T}^{\mathrm{T}} \mathrm{DT}
$$

with $:$ representing the matrix of elgenvectors of $V$. Thus, $V$ can be written

$$
V=\underset{\sim}{T} T^{T}
$$




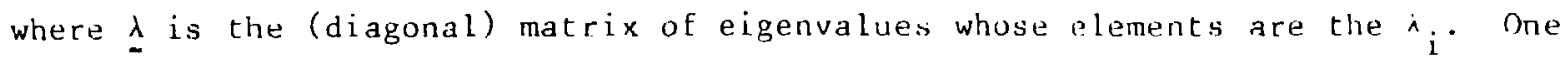
might infer from [1] that the espression given above for $A$ is essentially different from the one given by $(;)$, since nu connection is mentioned. Thus, it seems appropriate to demonstrate that the two are, in fact, equivalent, though the calculation is surprisingly lengthy.

To carry through the demonstration it is convenient to define a matrix $Y$ by

$$
Y=T^{T}[I \text { tr } V-V]^{-1} T
$$

which can be written explicitly as

$$
Y=\left(\begin{array}{ccc}
1 /\left(\lambda_{2}+\lambda_{3}\right) & 0 & 0 \\
0 & 1 !\left(\lambda_{1}+\lambda_{3}\right) & 0 \\
0 & 0 & 1 /\left(\lambda_{1}+\lambda_{2}\right) !
\end{array}\right)
$$

It is also convenient to define

$$
Q_{j k p q}=s_{i \ell} e_{j i k}{ }^{e} p \ell q
$$

with summation over $\&$ and $i$ implied, and $e_{i j k}$ representing the permutation symbol. Then ( 5 ) becomes, in index notation,

$$
A_{i k}=\frac{1}{2} Q_{i k p q} Z_{p q}
$$

with summation over $p$ and $q$ implied. Another useful quantity is

$$
B_{i j k}=T_{\ell i} e_{j \ell k}
$$

sumned on $\ell$. This makes it possible to wrice $Q$ in lhe form

$$
Q_{j k p q}=\sum_{i, \ell}^{B} B_{i j k}{ }^{\delta} i \ell^{Y} \ell^{B} \ell P q
$$


where the $Y_{\ell}$ are the diagonal elemeats of $Y$. Now, $Z$ can be expressed, using (26) as

$$
Z_{p q}=\sum_{r, t} T_{p r} \tilde{D}_{r t}\left(\lambda_{t}-\lambda_{r}\right) T_{q t} .
$$

When substituted into (30) a lengthy expression for A is obtained containing a group of terms which simplifies to the permutation symbol, viz

$$
\mathrm{B}_{\operatorname{spc}_{\mathrm{L}}} \mathrm{T}_{\mathrm{pr}} \mathrm{T}_{\mathrm{qt}_{\mathrm{t}}}=\mathrm{e}_{\mathrm{plq}} \mathrm{T}_{\mathrm{pr}} \mathrm{T} \mathrm{ss}_{\mathrm{qt}} \mathrm{T}_{\mathrm{rst}}
$$

Use of identities involving ptrmutation symbols given by Fiugge [17] are useful in these calculations. Combining (28)-(35) results, fiter some algebra, in

$$
A_{j k}=\frac{1}{2} \sum_{i} B_{i j k} Y_{i} h_{i}
$$

where

$$
h_{i}=\sum_{r, t} e_{i r t}\left(\lambda_{t}-i_{r}\right) \tilde{D}_{r t}
$$

This expression for A seems quite different from the one given by Nemat-Nasser, since it contains $\mathrm{T}$ linearly [in riew of (31)] whereas (24) zontains $\mathrm{T}$ quadratically. This dichotomy can be reconciled by noting that $T$ is orthogonal, a condition that can be expressed as

$$
\mathrm{T}_{\text {in }} \mathrm{e}_{i j k}=\mathrm{T}_{j \ell} \mathrm{T}_{\mathrm{km}} \mathrm{e}_{\ell \mathrm{mn}} \text {, }
$$

with summation on repeated subscripts. With this relation it can be shown that

$$
A_{j k}=\frac{1}{2} \sum_{r, t} \tilde{D}_{r t}\left(\lambda_{t}-\lambda_{r}\right) T_{j \ell} \ell^{T}{ }_{k m} \sum_{n} e_{l m r i} \because_{n} e_{n r t} .
$$

The sum over $n$ can be simplified by means of an identity given by Flugge as Eq. 3.20, whence 


$$
\sum_{n} Y_{n} e^{\ell m n} e_{n r t}=Y_{i i}\left(\delta_{r}^{l} \delta_{t}^{m}-\delta_{t}^{l} \delta_{r}^{m}\right)+\left(Y_{m}+Y_{r}\right)\left(\delta_{t}^{l} \delta_{r}^{m}-\delta_{r}^{l} \delta_{t}^{m}\right)
$$

summed on i. Substitution of this expression into (38) leads to

$$
A_{j k}=\frac{1}{2} \sum_{r, t}\left(\lambda_{t}-\lambda_{r}\right) \tilde{D}_{r t}\left(T T_{j} T_{k t}-T_{j t} T_{k r}\right)\left(Y_{i i}-Y_{t}-Y_{r}\right) .
$$

It can be verified by detailed enumeration that the last term on the right can be replaced by $l /\left(\lambda_{r}+\lambda_{t}\right)$ and that the two terms in the middle parentheses make equal contributions to the sum. Then (24) follows directly, completing the demonstration that it is equivalent to (5). However, the inversion method of (5) is computationally more efficient than the spectral method of (24), which requires extraction of the eigenvectors and eigenvalues of $V$. In fact, in special cases the matrix inversion can become quite simple, as illustrated by (16) for plane flows.

It is siated by Lee [2] that $\hat{\sigma}$, the polar stress rate, is not appropriate since "plasticity obeys an incremental or flow-type functional law, closer to a fluid than a solid cype, in wich the specific configuration of the initial undeformed state does not appear in the incremental or flow type constitutive relation at later times." This description fails to account for the physical basis of the constitutive law represented by (18) in its application to plasticity, which involves elastic-plastic behavior. Specifica?ly, in the Prandt-Reuss formulation the total stretching is represented as the sum of elastic and plastic parts, which can be expressed in matrix notation as

$$
D=D^{e}+D^{P}
$$

each of which has its own constitutive law. In a complete description of finite deformation theory $D^{e}$ would be related to the stress rate through a nonlinear relation guaranteeing elastic behavior, but for modest deformations it is suffi. cient to use a hypoelastic version of Hooke's law

$$
D^{e}=\dot{S} / 2 \mu+(\operatorname{tr} \dot{\sigma} / 9 k) I
$$


where $\mu$ and $k$ are elastic constants and $S$ is the deviator stress. The plastic strain rate is given by

$$
D^{t}=\lambda S
$$

Combining these equations we find

$$
D^{\prime}=\dot{3} / 2 \mu+\lambda S
$$

where $D^{\prime}$ is the deviator strain rate. Now, in the Prandt1-Reuss theory the stress lies on the yield surface

$$
\operatorname{tr} S^{2}=2 Y^{2}
$$

where $Y$ is the flow stress in simple shear. To determine $\lambda(44)$ is multiplied by $S$ and the trace taker, whence

$$
i=\operatorname{tr}\left(\sigma D^{\prime}\right) / 2 Y^{2}
$$

Rearranging theso equations and solving $f_{c} \cdot \dot{\sigma}$ leads to

$$
\dot{\sigma}=2 \mu(D-\lambda S)+\left(k-\frac{2}{3} \mu\right) I \operatorname{tr} D .
$$

The right-hand side is of the form indicated by (18), so that the complete constitucive law can be obtained by replacing $\dot{\sigma}$ on the left by $\hat{\sigma}$, as in (21). Thus, the presence of the stress rate arises from the elastic part of the constitutive law. Hence, the criticism of lee [2], that the derivation of (2l) is invalid for plastic flow because it involves the initial state, fails to account for the fact that the stress rate arises from the elastic part of the elastic-plastic model. Though the elastic part of $D$ is often small compared to the plastic part, it is important in several respects. From the computational point of view it allows the stress to vary smoothly in both space and time, for if the elastic part were dropped, the rigid-plastic behavior that ensued would be subject to discontinuities in stress following a reversal of strain rate, making numerical results 
poorly jehaved. From the physical point of view, the elastic part of the deformatior is vital in unloading behavior, and particularly in the wave structure behind shocks. Thus, though analysis of rigid-plastic behavior is useful in illusEra,jing relatively simple loading histories, as illustrated by Dafalias [3] in his analysis of kinemalıc hardening with large rutation, it prevents good accuraey in numerical simulations, which often involve complex loading and unloading paths.

\section{CONCLUDING REMARKS}

In conclusion, recent discussions maintaining that the stress rate derived in Ref. 8 (which I call polar stress rate here) is valid only for hypoelastic behavior, Lee [2], Dafalias [3], fail to consider in any detail the analysis given in Sec. IV of that paper, and summarized h re in the discussion of (21). This analysis is similar to those of Noll [7] and Green and MeTnnis [9]. It differs from Noll's in not setting $V=I$ and from Green and McInnis' in providing an exact, efficient expression for $A=\Omega-W$, given here as (5). That expression has been used successfully in numerical calculations following the outline given in Sec. VII of [8]. In a recent review Nemat-Nasser [1] provides an expression for A using Hill's spectral method, reproduced above as (24). Though apparently different from (5), it is shown here that they are equivalent. In that review it is stated that the various stress rates are equivalent, since they differ by terms that can be incorporated into the arbitrary function on the right representing material behavior. Another view is argued in this discussion, that the righthand side, representing behavior of a specific material in the absence of rotation, is fixed, and that the ordinary derivative of stress $\dot{\sigma}$ on the left of (18) has to be replaced by the polar rate $\hat{\sigma}$ to account for material rotation, leading to a unique representation. The polar stress rate is not different in character from any other tensor rate. For example, if strain is defined by

$$
E=\frac{1}{2}(B-I)
$$

it can be shown, using (3.36) of Ref. 8 , that

$$
\hat{E}=V D V
$$


where ( ) denotes the polar rate of (21). (A more detailed discussion of this rate is in preparation.) This polar strain rate accuunts for material rotation in a manrer analogous to stress rate. For modest deformation the choice of stress rate may not be important, and some believe that extended discussion is not warranted. Though this view is valid for many applications, in calculations involving severe distortion, such as shear banding and hypervelocity impact, a proper analysis is essential. Insofar as $I$ am aware, the importance of a suitable stress rate in connection with anisotropic materials and anisotropic microstructure has not been illustrated, but one can anticipate that the effect of material rotation plays a crucial role.

\section{REPERENCES}

1. S. Nemat-Nasser, "On Finite Plastic Flow of Crystaliine Solids and Geomaterials," J. Appl. Mech. Vol. 50, 1983, pp. 1114-1126.

2. E. H. Lee, "Elasto-plastic Deformat :on at Finite Strains," J. Appl. Mech., 1983, Vol. 50, PF. 554-560.

3. Y. F. Dafalias, "Corotational Rates for Kinematic Hardening at Large Plastic Deformations," J. Appl. Mech. Vol. 50, 1983, pp. 561-565.

4. J. C. Nagtegaal and J. E. de Jong, "Some Aspects of Non-isotropic Work Hardening in Finite Strain Plasticity," $P_{I} x$. of Workshop on Plasticity of Metals at Finite Strain, E. H. Lee and R. L. Mallet, eds, Stanford Univ., July 29-Aug. 1, 1981 .

5. S. Zaremba, "Sur une Forme perfectionee de la theorie de la relaxation," Bull. Int. Acad. Sci. Cracovie, 1903, Pp. 534-614.

6. G. Jaumann, "Geschlossenes System physikalischer und chemischer Differential gesetze," Sitzber. Akad. Wiss. Wien (IIa), 120, $19: 1$ PP. 385-530.

7. W. Noll, "On the Continuity of the Solid and Fluid States," J. Rat'l Mech. Anal., Vol. 4, 195j, PP. 3-81.

8. J. K. Dienes, "On the Analysis of Rotation and Stress Rate in Deforming Bodies," Acta Mechanica, Vol. 32, 1979, pp. 217-232.

9. A. E. Green and B. C. McInnis, "Generalized Hypoelasticity," Pros. Roy. Soc. Edinburgh A57, Part III, 1967, PP. 220-230.

10. R. Hill, "Aspects of Invariance in Solid Mechanics," Advances in Applied Mech. C.-S. Yih, ed., Vol. 18, 1978, PP. 1-75.

11. W. Prager, "An Elementary Discussion of Definitions of Stress Rate," Quarterly of Applied Math., Vol. 18, 1961, pp. 403-407. 
12. C. Truesdel1, The Elements of Continuum Mechanics, Springer-Verlag, 1966.

13. J. E. Marsden and T. J. R. Hughes, Mathematical Foundations of Elasticity, Prentice-Hall, 1983.

14. L. C. Woods, "The Bogus Axioms of Continuum Mechanics" Bull. IMA Vol. 17, 1981, pp. 98-102.

15. L. C. Woods, "Frame-indifferent Kinetic Theory," J. Fluid Mech. Vol. 136, 1983, pp. 423-433.

16. J. K. Dienes, "The Effect of Finite Rotation on a Problem in Plastic Deformation," Proc. Int'l Symposium on Plasticity, Bell Anniversary Volume, A. S. Khan, ed., 1984.

17. W. Flugge, Tensor Analysis and Continuum Mechanics, Springer-Verlag, 1972. 